\title{
The study of the longitude development of muons in air shower
}

\author{
Liping Wang, ${ }^{1,2, *}$ Lingling Ma, ${ }^{2,3}$ Cunfeng Feng ${ }^{1}$ and Huihai $\mathrm{He}^{2,3,4}$ \\ ${ }^{1}$ Shandong University, \\ 72, Binhai Road, Jimo, Qingdao, Shandong, China \\ ${ }^{2}$ The Institute of High Energy Physics of the Chinese Academy of Sciences, \\ 19B, Yuquan Road, Shijingshan District, Beijing, China \\ ${ }^{3}$ TIANFU Cosmic Ray Research Center, \\ Chengdu, Sichuan, China \\ ${ }^{4}$ University of Chinese Academy of Sciences, Department, \\ YuQuan Road.19A, shijingshan district, Beijing, China \\ E-mail: lpwang@mail.sdu.edu.cn, llma@ihep.ac.cn, \\ fengcfemail.sdu.edu.cn
}

The longitude development of the muonic component in the extensive air shower can help to determine mass composition of cosmic rays. By using the timing information of muons, the production positions of muons can be reconstructed. $X_{\max }^{\mu}$ which is the position with muon production reaching maximum is sensitive to the mass composition of cosmic rays. In this paper, the reconstruction of muon's geometrical production heights along the shower axis by using their timing information are studied for cosmic rays with energies from $1 \mathrm{PeV}$ to $10 \mathrm{PeV}$ by CORSIKA data. According to our analysis, the resolution of $X_{\text {max }}^{\mu}$ is $500 \mathrm{~g} / \mathrm{cm}^{2}$ with a resolution about 32 $\mathrm{g} / \mathrm{cm}^{2}$ for showers initiated by protons with energy $10 \mathrm{PeV}$, and zenith angle $\theta=45^{\circ}$.

$37^{\text {th }}$ International Cosmic Ray Conference (ICRC 2021)

July 12th - 23rd, 2021

Online - Berlin, Germany

\footnotetext{
${ }^{*}$ Presenter
} 


\section{INTRODUCTION}

The energy spectrum of cosmic rays covers more than ten magnitudes with a rapid decrease of its flux. Due to the low fluxes, the measurement of the energy spectrum of cosmic rays with energies above $100 \mathrm{TeV}$ can only be obtained indirectly by ground-based experiment by detecting the extensive air showers produced by interaction between cosmic rays and the Earth's atmosphere. However, the information of energies, mass compositions of primary cosmic rays are lost during the interactions. The reconstructions of energies, mass compositions are not only depended on the hadronic models used to described the development of air showers, but also depend on the composition models, due to the reconstructions of them can't be completely separated. In order to reduce the dependence, it is necessary to measure several parameters of air showers at the same time.According to the muon production and the longitude development of muons preserves the information of primary particles, the muon plays an important role in the study of composition identification with energy $10^{15} \mathrm{eV}-10^{16} \mathrm{eV}$.

The Pierre Auger experiment has achieved the reconstruction of the longitude development of muons by using the timing information recorded by their surface muon detectors for showers with energies above $10^{16} \mathrm{eV}$ [1]. In this paper, the production,propagation of muons in showers, the reconstruction method of muon's geometrical production heights along the shower axis are studied. Moreover, the effect of time resolution to the reconstruction are also studied by assuming time resolution $10 \mathrm{~ns} . X_{\max }^{\mu}$ which is the position with muon production reaching maximum can be obtained from the longitude development. The ability of $X_{\max }^{\mu}$ to identify the mass composition are also studied.

The paper is organised as follows. Sect 2 briefly describes the Corsika DATA we used .In Sect 3, the production, propagation of muons in showers, the method of muon's production position height are described in details. Sect 4 gives the muon profiles and discusses the influence for reconstruction accuracy caused by the distribution and time resolution of muon detector.Finally,in Sect 5 a brief summy is given together with some comments on the possible application of the method.

\section{SIMULATION DATA}

Corsika is the most popular software to simulate the development of air showers[2]. Corsika can record various properties of muons at the position of their origin such as energy,transverse momentum and produced height. In the simulations the EPOS-LHC hadronic interaction model for high energies and GHEISHA $\|-04$ for low energies are used. The altitude $(4410 \mathrm{~m})$ and magnetic field $(35 \mu \mathrm{T})$ are set according to the LHAASO site. So the vertical air depth is about $600 \mathrm{~g} / \mathrm{cm}^{2}$. In order to make sure the shower fully developed, the zenith angles of the showers are fixed as $45^{\circ}$, corresponding to air depth $850 \mathrm{~g} / \mathrm{cm}^{2}$ above the ground. In order to studied the mass compositions sensitivity of $X_{\text {max }}^{\mu}$, showers produced by protons and irons with three fixed energies $10^{15} \mathrm{eV}, 50^{15}$ $\mathrm{eV}$ and $10^{16} \mathrm{eV}$ are generated. 


\section{RECONSTRUCTION OF THE MUON PRODUCTION DEPTH}

\section{1 method of reconstructing the muon production depth.}

After the muons are produced in the air shower, they are mainly suffering the ionization energy loss during their propagation to the ground, so we can assume that muons travel in straight lines at the speed of light $\mathrm{c}$. With the assumptions, the production positions of the muons can be reconstructed according to the time delay corresponding to the time recorded near the shower core, as illustrated by Fig. 1. This method has been carried out by the Auger experiment[1] for the showers with energies above $10^{18} \mathrm{eV}$. According to the geometric relationship, the production positions of muons can be reconstructed by Equation 1.

$$
A O=\frac{1}{2}\left(\frac{O B^{\prime 2}}{c \times t_{g}}-c \times t_{g}\right)-c \times t_{\pi}
$$

where $t_{\pi}$ is correction due to the path traveled by the parent mesons and the average time delay $t_{\pi} 3 \mathrm{~ns}[3]$. Using the equation, and the atmosphere models, the air depth of the muon production position can be calculated.

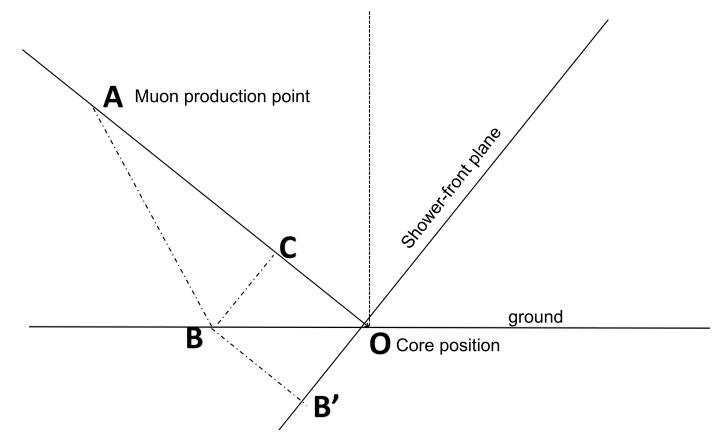

Figure 1: Coordinate system of the EAS according with CORSIKA,A = muon production point, $\mathrm{O}=$ shower core on ground, $\mathrm{B}=$ muon position on ground and $\mathrm{B}^{\prime}=$ muon position on shower front plane $\left(\mathrm{OB}^{\prime}\right)$

\subsection{Muon production in EAS and propagation through the atmosphere}

The method of reconstruction of muon production depth is based on two assumptions, one is that the muons are produced on the shower axis, the other one is the arriving directions of muons is the same as that when they are produced. The valid of the assumptions in the PeV energy range are studied in detail by Corsika data.Muons are weakly interacting particles and have correspondingly small interaction cross sections. Muons, though much less abundant than photons and electrons, play a very important role in air shower research. Since they are mainly subject to ionization losses only, energetic muons travel long distances in the atmosphere.It is argued that the transverse position of the production of muons, thus of the parent mesons decay, is confined to a relatively narrow cylinder[4]. Fig. 2(a) displays the distribution of the vertical distance from the production point of muons to the shower axis for protons and irons. According to Fig. 2(a), most production points of muons are confined in a cylinder with a radius of $10 \mathrm{~m}$. This distance is small compared with the size of EAS experiments, which span from hundreds of meters to several kilometers in the perpendicular plane. So muon production points can be approximately considered on the shower 
axis for muons with the distance from it to the shower core larger than $200 \mathrm{~m}$. During the muon's propagation to the ground, they also suffer multiple scattering, the deflection of magnetic field of the Earth, which can affect the directions of muons. The effect can be studied by the changes of muon directions from they are produced to they arrive at the ground. The distributions of the angular difference $\Delta$ are shown in Fig. 2(b) for protons and irons. The $\Delta$ s are mainly concentrated within $0.2^{\circ}$, so the influence from the magnetic field of the Earth and multiple scattering can be neglected and the trajectory of muons can be approximately considered as straight lines.

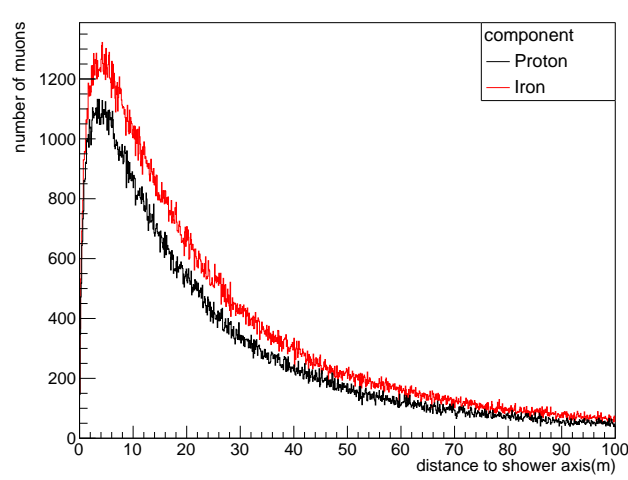

(a)

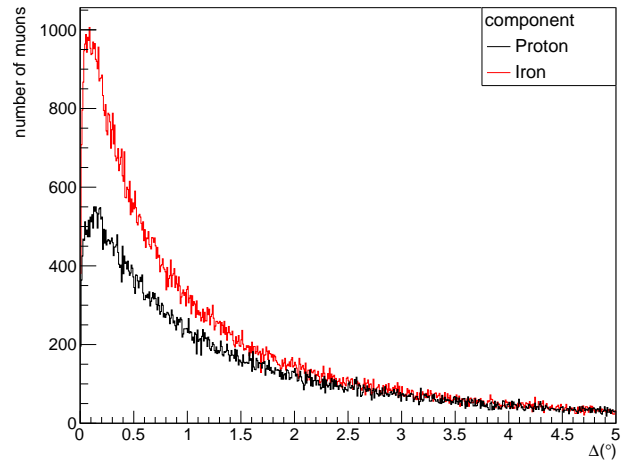

(b)

Figure 2: Fig2:(a): distributions of the perpendicular distance from the production point of muons to the shower axis for different primaries with energy $10^{16} \mathrm{eV}$ and zenith angle $45^{\circ}$. (b): The angular differences of muons when they are produced and when they are at the ground for different primaries with energy $10^{16} \mathrm{eV}$ and zenith angle $45^{\circ}$.

In the process of transmission,apart from the fact that muons are subject to geomagnetic deflection, an effect which is rapidly decreasing with increasing energy, muons are only exposed to ionization losses, occasional bremsstrahlung and to photonuclear reactions while propagating through the atmosphere.That means the total time delay of muons relative to the shower front plane not only geometric delay but also kinematic delay, geomagnetic delay and multiple scattering.The propagation of muons in matter below $1 \mathrm{TeV}$ is reviewed in[5].Figure 3 displays the different delays for different primaries, where the different contribution to the multiple scatering is included in $t_{\text {Rem }}$.At large distances from the core, the geometric delay is the most important. At distances typically from core to a few hundred meters, the kinematic delay has a large impact.In order to improve the accuracy of the reconstruction of the muon produced depth,the kinematic should be considered.After studying corsika data, kinematic delay distribution for different primaries and different energy can be fitted with an exponential function:

$$
t_{\varepsilon}=\exp \left(-0.5716+1.276 \times \log _{10}(r)\right)
$$

So,kinematic delay can be calculate from this formula,and then subtracted when reconstructed muon produced depth.

So it's know that muons mainly come from the decay of pions and kaons which are produced with a characteristic transverse momentum distribution inside a narrow cylinder around the shower 


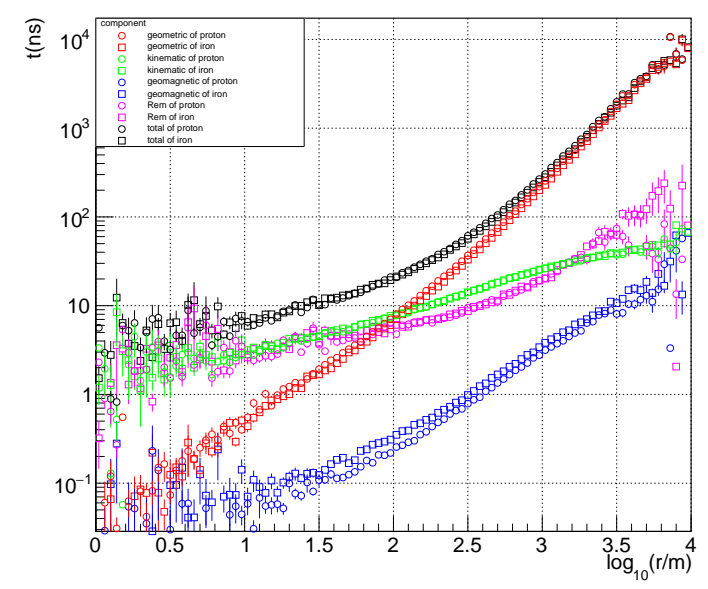

Figure 3: Average time delay for different contribution to the total delay for showers with energies with $10^{16}$ $\mathrm{eV}$ and zenith angle $45^{\circ}$ circles are for proton, squares are for iron.

axis.From figure 3, multiple scattering delay and geomagnetic delay account for $10 \%$ of the total time delay when $\mathrm{r}$ (distance from muon position on ground to core on SFP) $>400 \mathrm{~m}$, so multiple scattering effects and geomagnetic can be neglected.And it could thus be assumed that muons travel following straight lines, from the point where they are produced.

\section{FEATURES OF THE MUON PROFILES}

The true MPD distrubution,i.e. the one relative to all muons produced in air showers, cannot be reconstructed at ground level since a fraction of muons will decay before reaching the ground and muon with low energy can't trigger muon detector.On the other hand,by section3.2,it's easy to get that approximations can be valid when the distance from muon position on ground to core on $\mathrm{SFP}(\mathrm{r})>400 \mathrm{~m}$. So it is necessary to compare the distributions of all mouns produced depth and that of observed muons. In order to obtaining useful pgysical information from the MPD diatribution,for each shower we perform a fit of the muon longitudinal development profile with the Universal Shower Profile(USP) function, which describes well its non-symmetric shape[6].And the USP function is defined as

$$
\frac{d N}{d X}=\left(1+\frac{R}{L} \times\left(X-X_{\max }^{\mu}\right)\right)^{R^{-2}} \times \exp ^{-\frac{X-X_{\max }^{\mu}}{L R}},
$$

This function has three parameters, all related to the physics of the shower:the maximum of the profile $X_{\text {max }}^{\mu}$, the profile width L, and a parameter related to the distribution asymmetry R,which quantifies the deformation of the profile with respect to a Gaussian distribution. In this formula, the $\mathrm{N}$ refers to the number of produced muons and $\mathrm{X}$ is the depth expressed in $\mathrm{g} / \mathrm{cm}^{2}$.And the $X_{\max }^{\mu}$ accounts for the point along the shower axis where the production of muons reaches its maximum as the shower develps through the atmosphere. This parameter will be our main physical observable for composition and hadronic interactions studies. Figure 4(a) shown the muon profile of a shower 
initiated by a proton with energy with $10^{16} \mathrm{eV}$ and zenith angle $45^{\circ}$, it's obvious that the $X_{\max }^{\mu}$ of all mouns is closed to that of observed muons. Using the method provided by Sect. 3 and assuming time resolution $10 \mathrm{~ns}$ of muon detector can get distribution of muon reconstructed produced depth. As shown in figure 4(b), the reconstructed $X_{\max }^{\mu}$ is closed to the true $X_{\max }^{\mu}$.Increase the number of shower to study the $X_{\text {max }}^{\mu}$ distribution of different primaries under different energy.

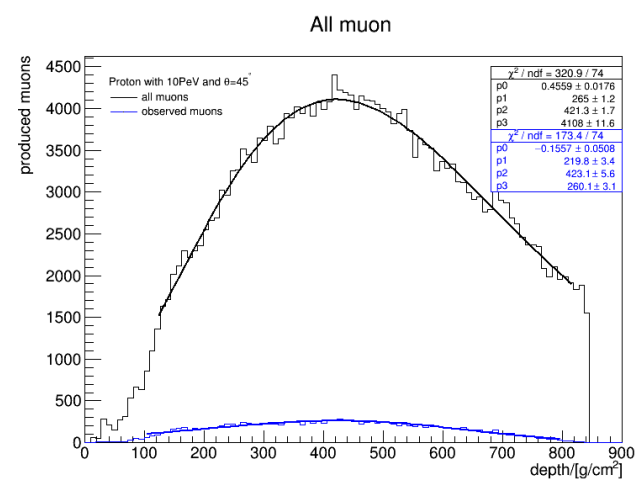

(a)

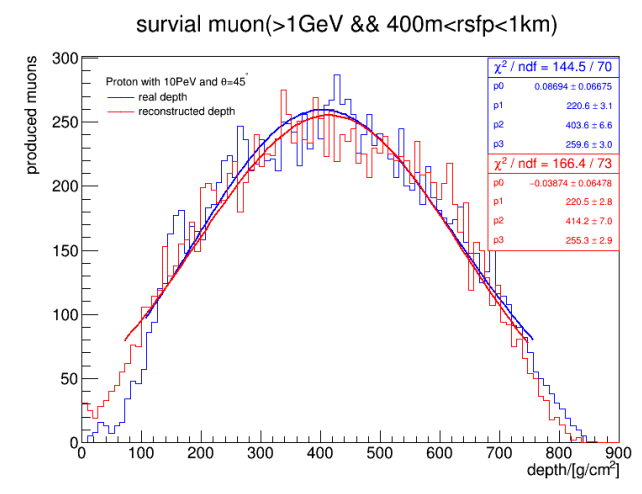

(b)

Figure 4: (a)The muon profile of a shower initiated by a proton with energy $10^{16} \mathrm{eV}$ and zenith angle $45^{\circ}$,black line indicates real produced depth distribution of all muons, bule line indicates real produced depth distribution of observed muons.(b)The muon profile of a shower initiated by a proton with energy $10^{16} \mathrm{eV}$ and zenith angle $45^{\circ}$,blue line indicates real produced depth distribution of observed muons, red line indicates reconstructed produced depth distribution of observed muons

In addition to considering the time resolution, the distribution of muon detectors should also be considered. In this paper data analysis is based on a detector array with detector separation of $30 \mathrm{~m}$, similar with the MD detector of Large High Attitude Air Shower Observation(LHAASO).Figure 5(a) shows muon distribution and black point is distribution of muons which can be detected. Figure5(b) shows distribution of muons reconstructed produced depth and USP function also could fit this distribution. Figure 6 left compares the distribution of true $X_{\max }^{\mu}$ and distribution of reconstructed $X_{\max }^{\mu}$ which considering the time resolution and distribution of muon detector.So it's easy to get that for proton with energy $=10^{16} \mathrm{eV}$ and zenith $=45^{\circ}$, the $X_{\max }^{\mu}$ is $500 \mathrm{~g} / \mathrm{cm}^{2}$ with a resolution about $32 \mathrm{~g} / \mathrm{cm}^{2}$. Compare the reconstructed $X_{\max }^{\mu}$ which only considering time resolution, after considering muons detector distribution makes the bias worse.Now there is question that need to be studied. Whether the reconstructed $X_{\text {max }}^{\mu}$ can still identify the mass compositions of cosmic rays? After the reconstruction of the longitudinal muon profile with considering time resolution and distribution of muon detectors, the maximum of the distribution $X_{\max }^{\mu}$ is obtained by fitting the profile with the USP function. After compare $X_{m}^{\mu} a x$ of proton and iron, it's easy to get that reconstructed $X_{\text {max }}^{\mu}$ is sensitive to the nature of the primary particles and can be used to identify mass compositions of cosmic rays. 


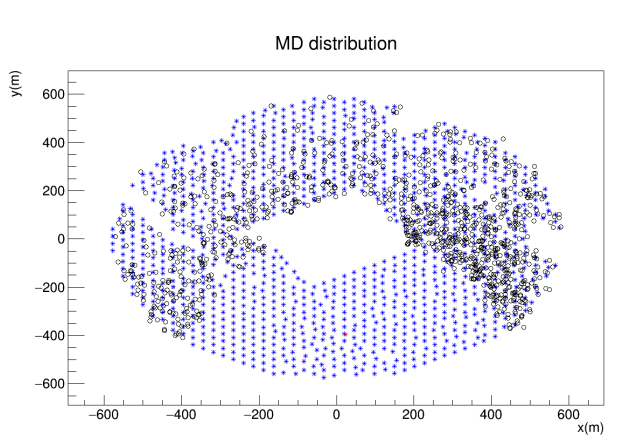

(a)

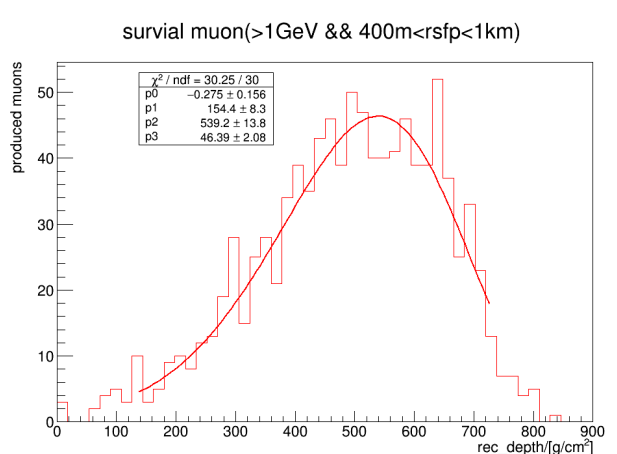

(b)

Figure 5: (a) Distribution of muons on MD detectors;blue point is distribution of muon detector, red star is core position, black circle is muon which can be detected by muon detector.(b) distribution of muons which can be detected reconstrcted depth.
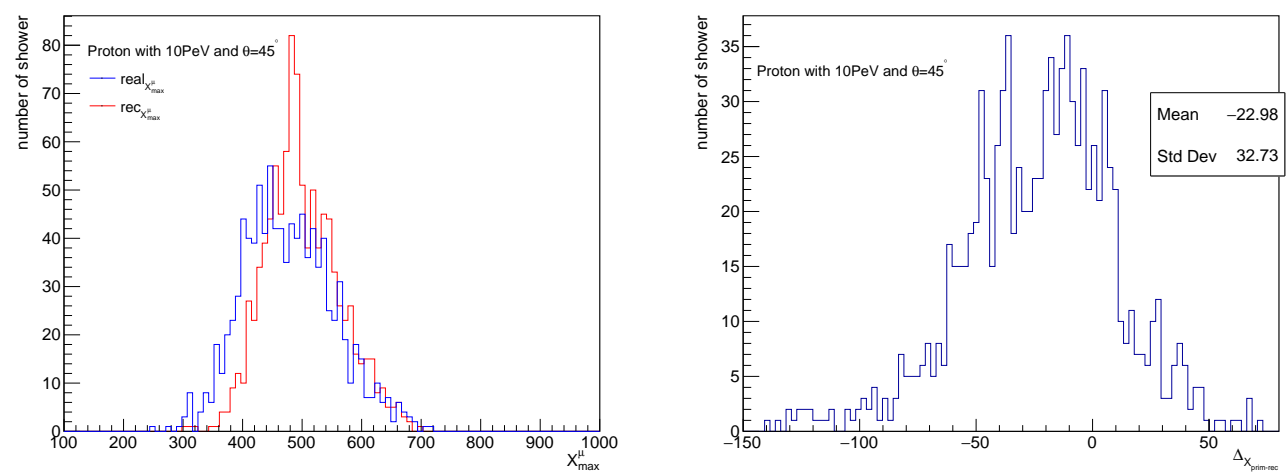

Figure 6: (Left) real $X_{\text {max }}^{\mu}$ and reconstructed $X_{\text {max }}^{\mu}$ distributions initiated by protons with energy $10^{16} \mathrm{eV}$ and zenith angle $45^{\circ}$ (Right) :distribution of $r e a l_{X_{\max }^{\mu}}-r e c_{X_{\max }^{\mu}}$ according to the left panel.

\section{Summary}

In this work, using CORSIKA simulations, the muon production depth in the air shower is reconstructed according the geometry effect.This model which relates the arrival time of muons at the ground with their depths is valid for nenrgy with $10^{15} \mathrm{eV}-10^{16} \mathrm{eV}$.The maximum of the muon production depth distribution, $X_{\max }^{\mu}$ can be obtained by fitting the longitudinal muon profile with the Universal Shower Profile function. And it's $500 \mathrm{~g} / \mathrm{cm}^{2}$ with a resolution about $32 \mathrm{~g} / \mathrm{cm}^{2}$ for proton with energy $=10^{16} \mathrm{eV}$ and zenith angle $\theta=45^{\circ}$.

\section{References}

[1] A. Aab et al. (Pierre Auger), Phys. Rev. D 90, 012012 (2014), [Addendum: Phys.Rev.D 90, 039904 (2014), Erratum: Phys.Rev.D 92, 019903 (2015)], arXiv:1407.5919 [hep-ex] . 
[2] D. Heck, J. Knapp, J. N. Capdevielle, G. Schatz, and T. Thouw, Corsika: A monte carlo code to simulate extensive air showers (1998).

[3] L. Cazon, R. A. Vazquez, and E. Zas, Astropart. Phys. 23, 393 (2005), arXiv:astro-ph/0412338

[4] L. Cazon, R. Conceicao, M. Pimenta, and E. Santos, Astropart. Phys. 36, 211 (2012), arXiv:1201.5294 [astro-ph.HE] .

[5] K. Nakamura et al. (Particle Data Group), J. Phys. G 37, 075021 (2010).

[6] S. Andringa, L. Cazon, R. Conceicao, and M. Pimenta, Astropart. Phys. 35, 821 (2012), arXiv:1111.1424 [hep-ph] .

[7] J. R. Hörandel, in Neutrinos and Explosive Events in the Universe, edited by M. M. Shapiro, T. Stanev, and J. P. Wefel (Springer Netherlands, Dordrecht, 2005) pp. 365-376.

[8] L. Collica (Pierre Auger), Eur. Phys. J. Plus 131, 301 (2016), arXiv:1609.02498 [astro-ph.HE]

[9] X. Bai et al., arXiv:1905.02773 [astro-ph.HE] (2019).

[10] S. Cui, Y. Liu, Y. Liu, and X. Ma (LHAASO), Astropart. Phys. 54, 86 (2014).

[11] Z. Cao et al., Nature 594, 33 (2021).

[12] D. Heck and T. Pierog, Extensive air shower simulation with corsika: A user's manual (version 6.7) (2007). 